\title{
Geometric optics with critical vanishing viscosity for one-dimensional semilinear initial value problems
}

Stéphane Junca

\begin{abstract}
We study the propagation of high frequency oscillations for one dimensional semi-linear hyperbolic systems with small parabolic perturbations. We obtain a new degenerate parabolic system for the profile, and valid an asymptotic development in the spirit of Joly, Métivier and Rauch.
\end{abstract}

\section{Introduction}

In [13], Joly, Métivier and Rauch gave a rigorous description of solutions to one dimensional nonlinear hyperbolic equations with smooth and highly oscillatory initial data. On the other hand, numerous physical problems involve parabolic partial differential equations with small viscosity, for instance the Navier-Stokes system with large Reynolds number. It is then of interest to implement the Joly, Métivier, Rauch framework with a small diffusion. The first step to study such multiphase expansions is the semilinear case. Thus, we wish to investigate the propagation of high frequency oscillations for the following nonlinear parabolic system with highly oscillatory initial data $u_{0}^{\varepsilon}$ and small positive viscosity $\nu$ :

$$
\begin{aligned}
-\nu \frac{\partial^{2} u^{\varepsilon}}{\partial x^{2}}+\frac{\partial u^{\varepsilon}}{\partial t}+A(t, x) \frac{\partial u^{\varepsilon}}{\partial x} & =F\left(t, x, u^{\varepsilon}\right) \\
u^{\varepsilon}(0, x) & =u_{0}^{\varepsilon}(x) .
\end{aligned}
$$

2000 Mathematics Subject Classification: Primary: 35K45, 35L60; Secondary: 35B25.

Keywords: Nonlinear geometric optics, small viscosity, profile, phase, non stationary phase, maximum principle, energy estimates, interpolation, weakly coupled parabolic systems. 
The matrix $A(t, x)$ is a smooth $N \times N$ real matrix with $N$ distinct real eigenvalues: $\lambda_{1}(t, x)<\lambda_{2}(t, x)<\cdots<\lambda_{N}(t, x)$, in such a way that $\partial_{t}+$ $A(t, x) \partial_{x}$ is a strictly hyperbolic operator.

In this paper, we will focus our attention on the special case of rapidly oscillating data with a given vector phase $\varphi^{0}(x)$, frequency $1 / \varepsilon$ and a viscosity which is equal to the square of the wavelength:

$$
\begin{aligned}
u^{\varepsilon}(0, x)=u_{0}^{\varepsilon}(x) & =U_{0}\left(x, \frac{\varphi^{0}(x)}{\varepsilon}\right), \\
\nu & =\varepsilon^{2}
\end{aligned}
$$

Therefore, with such oscillating data, the viscous coefficient has critical size (see next section), so that we expect to have $\varepsilon^{2} \partial_{x}^{2} u^{\varepsilon}$ of order one. And we hope to see interactions of high oscillations with the viscous term. Indeed, it is the aim of this paper to justify a geometric optics expansion:

$$
u^{\varepsilon}(t, x)=U\left(t, x, \frac{\varphi(t, x)}{\varepsilon}\right)+o(1),
$$

where $\varphi$ is a vector phase as in the hyperbolic case (without viscosity) and the profile $U$ satisfies a new degenerate parabolic modulation system. With such diagonal viscosity, we validate WKB expansion in $L_{x}^{2}$ with initial data only bounded in $L_{x}^{2} \cap L_{x}^{\infty}$.

There is a rich literature devoted to nonlinear geometric optics expansions. The classical work here is [13]. For the quasilinear case restricted to constant background states and linear phases, see [4, 5, 11, 12, 15, 16, 18]. For the semilinear case, see $[13,14,20,21]$. For some results with oscillations and viscosity in multidimensional framework with one phase see $[1,7,8]$, with a nonlinear instability result obtained trough WKB expansions in the second reference. To a reader interested by an overview on nonlinear geometric optics we recommend the survey paper [6] and references therein.

The paper is organized as follows. In short section 2 we briefly explain the choice of viscosity (1.4) on a simple example. In section 3 we collect relevant material from [13], give new parabolic profile systems and state our main results. Section 4 set up the notations for the almost diagonal system, and reviews some of the standard estimates on parabolic equations. We conclude by a useful interpolation lemma. In section 5, we prove uniform existence in time for solutions of (1.1), (1.4) with initial data (1.2) uniformly bounded in $L^{\infty} \cap L^{2}$. Section 6 is devoted to the study of the profile system. Section 7 deals with the important linear scalar case. Then, in section 8 , geometric optics expansion is validated. Finally, in section 9, we give a few comments and comparisons with the inviscid case. 


\section{About viscosity size}

Let us examine a simple example to get critical size of viscosity $\nu$ with $\varepsilon$-wavelength:

$$
\partial_{t} u^{\varepsilon}+\lambda \partial_{x} u^{\varepsilon}=\nu \partial_{x}^{2} u^{\varepsilon}, \quad u^{\varepsilon}(0, x)=u_{0}(x / \varepsilon),
$$

where $\nu>0, u_{0}$ is a smooth one-periodic function with zero mean. We have the classical decay rate in $L^{\infty}\left(\mathbb{R}_{x}\right)$ for one-periodic solutions with zero mean:

$$
\left\|u^{\varepsilon}(t, .)\right\|_{\infty} \leq \frac{\left\|u_{0}\right\|_{\infty}}{\sqrt{\pi t}} \frac{\varepsilon}{\sqrt{\nu}}
$$

If $\nu \gg \varepsilon^{2}$ then diffusion is too strong and kills oscillations.

Now, let $v^{\varepsilon}(t, x)=u^{\varepsilon}(t, x)-u_{0}\left(\frac{x-\lambda t}{\varepsilon}\right)$. $v^{\varepsilon}$ satisfies the following Cauchy problem:

$$
\partial_{t} v^{\varepsilon}+\lambda \partial_{x} v^{\varepsilon}-\nu \partial_{x}^{2} v^{\varepsilon}=\nu \varepsilon^{-2} u_{0}^{\prime \prime}, \quad v^{\varepsilon}(0, x)=0 .
$$

Using the maximum principle for $v^{\varepsilon}$ we conclude that:

$$
\left\|u^{\varepsilon}(t, x)-u_{0}\left(\frac{x-\lambda t}{\varepsilon}\right)\right\|_{\infty} \leq t\left\|u_{0}^{\prime \prime}\right\|_{\infty} \frac{\nu}{\varepsilon^{2}}
$$

Thus, if $\nu \ll \varepsilon^{2}$,we have the same hyperbolic behavior as when $\nu=0$. Thus, the critical viscosity size is $\nu \sim \varepsilon^{2}$. Furthermore, for $\nu=\varepsilon^{2}$, inequalities (2.2), (2.3) give bounds of order one and we get an exact geometric optics expansion and a parabolic profile equation:

$$
u^{\varepsilon}(t, x)=U\left(t, \frac{x-\lambda t}{\varepsilon}\right), \quad \text { where }-\partial_{\theta}^{2} U+\partial_{t} U=0, \quad U(0, \theta)=u_{0}(\theta) .
$$

\section{Main Results}

The viscosity $\nu$ is now fixed by (1.4). Our first goal is to prove that the exact solutions of (1.1), (1.2) exist on a domain independent of $\varepsilon \in\left(0, \varepsilon_{0}\right]$ for a positive $\varepsilon_{0}$. Let us begin with fixing an arbitrary $T_{0}>0$ for all the sequel. For such parabolic systems, in order to avoid boundary value problems, we will work globally in the space variable and assume that the entries of matrix

$$
A(t, x), \partial_{x} A(t, x), \partial_{x}^{2} A(t, x) \text { are bounded in } L^{\infty}\left(\left[0, T_{0}\right] \times \mathbb{R}_{x}\right) .
$$

Similarly, $F(t, x, u)$ is a smooth non linear function such that, for any compact $K \subset \mathbb{R}_{u}^{N}$,

$$
F(t, x, u), \partial_{u} F(t, x, u) \text { are bounded in } L^{\infty}\left(\left[0, T_{0}\right] \times \mathbb{R}_{x} \times K_{u}\right) .
$$


Furthermore, to have energy estimates, we also assume that

$$
F(t, x, 0) \in C^{0}\left(\left[0, T_{0}\right] ; L^{2}\left(\mathbb{R}_{x}, \mathbb{R}^{N}\right)\right) .
$$

The following preliminary result gives sufficient conditions on the Cauchy data $u_{\varepsilon}^{0}$ to get existence of a solution on a domain independent of $\varepsilon$.

Proposition 3.1. (Uniform existence) Under assumptions (3.1), (3.2) and (3.3), if the family $\left(u_{0}^{\varepsilon}\right)_{0<\varepsilon \leq 1}$ is bounded in $L^{\infty} \cap L^{2}\left(\mathbb{R}, \mathbb{R}^{N}\right)$, then, there exist $T>0$ and $\varepsilon_{0}>0$ such that, for any $\varepsilon \in\left(0, \varepsilon_{0}\right]$, the system (1.1), (1.2), (1.4) has a unique weak solution $u^{\varepsilon}$ in $E:=C\left([0, T] ; L^{2}\left(\mathbb{R}, \mathbb{R}^{N}\right)\right) \cap$ $L^{\infty}\left([0, T] \times \mathbb{R}, \mathbb{R}^{N}\right)$. Furthermore the family $\left(u^{\varepsilon}\right)_{0<\varepsilon \leq 1}$ is uniformly bounded in $E$ independently of $\varepsilon$.

The proof of this result is given in section 5 and uses energy estimates for the linearized problem together with the maximum principal for scalar parabolic equations and an interpolation estimate.

After this preliminary result, we are going to find phases and the profile system. Typically in $(1.3), \varphi^{0} \in C^{\infty}\left(\mathbb{R}, \mathbb{R}^{\mathrm{m}}\right)$ and $U_{0}(x, \theta)$ is smooth function, supported in $\{|x|<1\}$ and $\mathbb{Z}^{\mathrm{m}}$-periodic with respect to $\theta \in \Theta^{0}=\mathbb{R}^{\mathrm{m}}$, but we will also consider more general almost-periodic profiles as in [13]. In order to state the main result, we have to introduce some notations.

\section{Phases and characteristic vector fields:}

We note $\varphi^{0}=\left(\varphi_{1}^{0}, \ldots, \varphi_{\mathrm{m}}^{0}\right)$ where $\varphi_{j}^{0} \in C^{\infty}(\mathbb{R} ; \mathbb{R})$, and assume that the $\varphi_{1}^{0}, \ldots, \varphi_{\mathrm{m}}^{0}$ are linearly independent. As in a pure hyperbolic problem, we use characteristic vector fields of the hyperbolic operator $\partial_{t}+A(t, x) \partial_{x}$

$$
X_{j}=\partial_{t}+\lambda_{j}(t, x) \partial_{x}, j=1, \ldots, N
$$

and define the (vector valued) phases $\varphi_{j} \in C^{\infty}\left(\left[0, T_{0}\right], \times \mathbb{R} ; \mathbb{R}^{\mathrm{m}}\right), j=1, \ldots, N$ by

$$
X_{j} \varphi_{j}=0, \quad \varphi_{j}(0, x)=\varphi^{0}(x) .
$$

We then call $\varphi=\left(\varphi_{1}, \ldots, \varphi_{N}\right) \in C^{\infty}\left(\left[0, T_{0}\right] \times \mathbb{R} ;\left(\mathbb{R}^{\mathrm{m}}\right)^{N}\right)$ and introduce the following two linear spaces:

$$
\Theta^{0}:=\operatorname{span}\left\{\varphi^{0}(\mathbb{R})\right\}=\mathbb{R}^{\mathrm{m}}, \quad \Theta:=\operatorname{span}\left\{\varphi\left(\left[0, T_{0}\right] \times \mathbb{R}\right)\right\} \subset \mathbb{R}^{\mathrm{m} N} .
$$

First equality $\Theta^{0}=\mathbb{R}^{\mathrm{m}}$ comes from linear independence of $\varphi_{j}^{0}, j=1, \ldots, \mathrm{m}$. Strict inequality $\operatorname{dim}(\Theta)<N \mathrm{~m}$ holds if and only if there exists resonance relations between the phases $\varphi_{j}$, i.e.:

$$
R:=\left\{\alpha \in \mathbb{R}^{N} ; \alpha . \varphi \equiv 0 \text { on }\left[0, T_{0}\right] \times \mathbb{R}\right\} \neq\{0\} .
$$

Notice that $R^{\perp}=\Theta$. 
Let's call $\theta=\left(\theta_{1}, \ldots, \theta_{N}\right)$ the independent variables in the space $\mathbb{R}^{\mathrm{m}} \times$ $\cdots \times \mathbb{R}^{\mathrm{m}}$, where $\theta_{j}=\left(\theta_{j, 1}, \ldots, \theta_{j, \mathrm{~m}}\right) \in \mathbb{R}^{\mathrm{m}}$. Let $\Pi_{j}$ be the linear projection on $j$-component: $\Pi_{j}: \Theta \rightarrow \mathbb{R}^{\mathrm{m}}, \Pi_{j}(\theta)=\theta_{j}$. Let us define $\Psi_{j}=\operatorname{ker} \Pi_{j} \cap \Theta$ and $\Theta_{j}:=\Psi_{j}^{\perp} \cap \Theta$.

Now, to valid a geometric optics expansion, we need some assumptions on phases. We use the more general transversality conditions from [13] (named precisely weak transversality condition in [13]):

$$
\left\{\begin{array}{l}
\text { either } X_{j}(\alpha . \varphi) \equiv 0 \text { on }\left[0, T_{0}\right] \times \mathbb{R} \\
\text { or } X_{j}(\alpha . \varphi) \neq 0 \text { almost everywhere on }\left[0, T_{0}\right] \times \mathbb{R}
\end{array}\right.
$$

In second case of (3.6), $\alpha . \varphi$ is said to be transverse to $X_{j}$. The transverse phases case is realized when (3.6) is satisfied for all $\alpha$ in $\Theta^{*}$ and for all $j$.

To avoid constant phases, we assume non stationary condition on initial phases:

$$
\forall \beta \in \mathbb{R}^{\mathrm{m}}, \partial_{x}\left(\beta . \varphi^{0}\right) \neq 0 \text { almost everywhere on } \mathbb{R} \text {. }
$$

Notice that (see [13]) such conditions (3.6) and (3.7) avoid constant phases.

We also assume following closedness property for all $\alpha$ in $\Theta^{*}$ and for all $j$ :

$$
X_{j}(\alpha . \varphi) \equiv 0 \text { on }\left[0, T_{0}\right] \times \mathbb{R} \Rightarrow \exists \beta \in \mathbb{R}^{\mathrm{m}} \text {, such that } \alpha . \varphi \equiv \beta . \varphi_{j} .
$$

Indeed, it is not a restrictive assumption, since, without loss of generality, we can add new phases coming from resonance as new initial phases in $\varphi^{0}$. Thus, we increase $\mathrm{m}$, the number of initial phases.

\section{Almost periodic profiles:}

As in classical geometrical optics ([13]), one is naturally led to work within the class of almost periodic functions. If $Z$ is some finite dimensional real linear space, we call $C_{a p}^{0}(Z, \mathbb{R})$ the topological closure in $L^{\infty}(Z, \mathbb{R})$ of the linear subspace $\operatorname{span}\left\{\xi \mapsto \exp (\iota \alpha . \xi), \alpha \in Z^{*}\right\}$. We also denote $C_{a p}^{\infty}(Z, \mathbb{R})=$ $C_{a p}^{0}(Z, \mathbb{R}) \cap C^{\infty}(Z, \mathbb{R})$.

\section{Averaging operators:}

We use the averaging operators already introduced by [13] which are defined as follows. Let $\mu_{j}$ be the Lebesgue measure on $\Psi_{j}, Q_{j}$ a cubic subset of $\Psi_{j}$ such that $\mu_{j}\left(Q_{j}\right)=1$. We define the averaging operator $\mathbb{E}_{j}$ acting on $C_{a p}^{0}(\Theta, \mathbb{R})$, by the formula

$$
\left(\mathbb{E}_{j} v\right)\left(\theta_{j}\right)=\lim _{T \rightarrow+\infty} \frac{1}{T^{\operatorname{dim} \Psi_{j}}} \int_{T \cdot Q_{j}} v\left(\theta_{j}+\xi\right) d \mu_{j}(\xi)
$$


In the previous equation the right hand side actually only depends on the variable $\theta_{j}$ and

$$
\mathbb{E}_{j}: C_{a p}^{0}(\Theta, \mathbb{R}) \longrightarrow C_{a p}^{0}\left(\Theta_{j}, \mathbb{R}\right)
$$

is a continuous linear projector. This map can be also defined on elementary exponential functions by the following rule

$$
\mathbb{E}_{j}(\exp (i \alpha . \theta))=\left\{\begin{array}{cl}
\exp (i \alpha . \theta) & \text { if } X_{j}(\alpha \cdot \varphi)=0 \\
0 & \text { else }
\end{array}\right.
$$

and then extended by density and continuity (cf. [13]).

\section{The profile system:}

Let $\Lambda(t, x)$ be the diagonal matrix with components $\left(\lambda_{1}(t, x), \ldots, \lambda_{N}(t, x)\right)$. There exists a smooth invertible matrix $P(t, x)$ such that: $P^{-1} A P=\Lambda$. To get the profile system, it suffices to perform a WKB expansion for $v^{\varepsilon}(t, x)=$ $P^{-1}(t, x) u^{\varepsilon}(t, x)$ with the anzatz:

$$
v^{\varepsilon}(t, x)=V\left(t, x, \frac{\varphi(t, x)}{\varepsilon}\right)+o(1) .
$$

In this way, we formally obtain the differential operator $\mathcal{D}_{j}$

$$
\mathcal{D}_{j}=\sum_{k=1}^{\mathrm{m}} \frac{\partial \varphi_{j k}}{\partial x} \frac{\partial}{\partial \theta_{j k}}=\partial_{x} \varphi_{j} \cdot \nabla_{\theta_{j}} .
$$

We also obtain the initial profile $V_{0}$ and the new right hand side of (1.1) written in the new base where $A$ is a diagonal matrix. We give profile equations in the next proposition.

$$
\begin{aligned}
V_{0}(x, \theta) & =P^{-1}(0, x) U_{0}(x, \theta), \\
G_{P}(t, x, v) & =P^{-1}(t, x)\left(\partial_{t} P(t, x)+\Lambda(t, x) \partial_{x} P(t, x)\right) v \\
G(t, x, v) & =P^{-1}(t, x) F(t, x, P(t, x) v)-G_{P}(t, x, v) .
\end{aligned}
$$

Proposition 3.2. (Equations and existence of profile) Under assumptions (3.1), (3.2), (3.3), and if $U_{0}$ belongs in $L_{x}^{2}\left(\mathbb{R} ; C_{a p}^{0}\left(\Theta^{0}, \mathbb{R}^{N}\right)\right) \cap$ $L^{\infty}\left(\mathbb{R}_{x} \times \Theta^{0}, \mathbb{R}^{N}\right)$, there exists a positive $T$ such that, following system, with $j=1, \ldots, N$, admits a unique solution in $C^{0}\left([0, T] ; L_{x}^{2}\left(\mathbb{R} ; C_{a p}^{0}\left(\Theta, \mathbb{R}^{N}\right)\right)\right) \cap$ $L^{\infty}\left([0, T] \times \mathbb{R} \times \Theta, \mathbb{R}^{N}\right):$

$$
\begin{aligned}
-\mathcal{D}_{j}^{2} V_{j}+X_{j} V_{j} & =\mathbb{E}_{j} G_{j}(t, x, V), \\
V_{j}(0, x, \theta) & =V_{0, j}\left(x, \theta_{j}\right) .
\end{aligned}
$$


Indeed we have $V_{j}(t, x, \theta)=V_{j}\left(t, x, \theta_{j}\right)$. We set $U(t, x, \theta)=P(t, x) V(t, x, \theta)$. We can now state the main result of the paper which describes the propagation of the oscillations for the parabolic system.

Theorem 3.1. (Validity of the geometric optics expansion) We assume that $U_{0}$ belongs to $L^{2}\left(\mathbb{R}_{x} ; C_{a p}^{0}\left(\mathbb{R}^{\mathrm{m}}, \mathbb{R}^{N}\right)\right) \cap L^{\infty}\left(\mathbb{R}_{x} \times \mathbb{R}^{\mathrm{m}}, \mathbb{R}^{N}\right)$ and assumptions (3.1), (3.2), (3.3) are satisfied.

Then there exist $T>0, \varepsilon_{0}>0$ such that, for any $\varepsilon \in\left(0, \varepsilon_{0}\right]$, the system (1.1), (1.4) with initial data (1.3) admits an unique solution $u^{\varepsilon}$ in $C\left([0, T] ; L^{2}\left(\mathbb{R}, \mathbb{R}^{N}\right)\right) \cap L^{\infty}\left([0, T] \times \mathbb{R}, \mathbb{R}^{N}\right)$, and the system (3.13), (3.14) admits an unique solution $U$ in $C^{0}\left([0, T] ; L_{x}^{2}\left(\mathbb{R} ; C_{a p}^{0}\left(\Theta, \mathbb{R}^{N}\right)\right)\right) \cap L^{\infty}([0, T] \times$ $\left.\mathbb{R} \times \Theta, \mathbb{R}^{N}\right)$.

Furthermore, if phases are transverse, we have the following geometric optics expansion:

$$
\lim _{\varepsilon \rightarrow 0}\left[u^{\varepsilon}(t, x)-U\left(t, x, \frac{\varphi(t, x)}{\varepsilon}\right)\right]=0 \quad \text { in } \quad L^{\infty}\left([0, T] ; L^{2}\left(\mathbb{R}, \mathbb{R}^{N}\right)\right) .
$$

\section{Almost diagonal system, $L^{\infty}$ and $L^{2}$ estimates}

We diagonalize $A$. We then write system (1.1), (1.4), in this new base. Later we recall and give energy and uniform estimates used below.

\section{The almost diagonal system:}

We diagonalize the hyperbolic operator $\partial_{t}+A(t, x) \partial_{x}$ in system (1.1). Unfortunately, this procedure slightly couples first order derivatives. We will however control this coupling effect with Lemma 4.1 in Section 5. More precisely, let us give following notations.

$$
\begin{aligned}
v^{\varepsilon}(t, x) & =P^{-1}(t, x) u^{\varepsilon}(t, x), \quad v_{0}^{\varepsilon}(x):=P^{-1}(t, x) u_{0}^{\varepsilon}(t, x), \\
Q_{1}(t, x) & =2 P^{-1}(t, x) \partial_{x} P(t, x), \\
Q_{2}(t, x) & =P^{-1}(t, x) \partial_{x}^{2} P(t, x), \\
G^{\varepsilon}(t, x, v) & =G(t, x, v)+\varepsilon^{2} Q_{2}(t, x) v, \\
\mathbb{L}^{\varepsilon} & =-\varepsilon^{2} \frac{\partial^{2}}{\partial x^{2}}-\varepsilon^{2} Q_{1}(t, x) \frac{\partial}{\partial x}+\frac{\partial}{\partial t}+\Lambda(t, x) \frac{\partial}{\partial x}
\end{aligned}
$$

$v^{\varepsilon}$ is the solution of the almost diagonal system with initial data

$$
\mathbb{L}^{\varepsilon} v^{\varepsilon}=G^{\varepsilon}\left(t, x, v^{\varepsilon}\right), \quad v^{\varepsilon}(0, x)=v_{0}^{\varepsilon}(x) .
$$

We use below the diagonal part of the linear operator $\mathbb{L}^{\varepsilon}$ :

$$
\mathbb{D}^{\varepsilon}=\mathbb{L}^{\varepsilon}+\varepsilon^{2} Q_{1} \frac{\partial}{\partial x}=-\varepsilon^{2} \frac{\partial^{2}}{\partial x^{2}}+\frac{\partial}{\partial t}+\Lambda(t, x) \frac{\partial}{\partial x}
$$




\section{Energy and $L^{\infty}$ estimates:}

First notice that $\Lambda, P$ and $P^{-1}$ also satisfy assumption (3.1). So $\partial_{x} \Lambda, \partial_{x} Q_{1}$ are bounded on $\left[0, T_{0}\right] \times \mathbb{R}$ and we have:

Theorem 4.1. (Classical energy estimates) Let $1 \geq \nu>0, T_{0} \geq T>0$, $L$ and $Q_{1}$ bounded matrix on $\left[0, T_{0}\right] \times \mathbb{R}, w(t, x)$ the unique solution in $C^{0}\left(\left[0, T_{0}\right], L^{2}\left(\mathbb{R}_{x}, \mathbb{R}^{N}\right)\right)$ of:

$$
\begin{aligned}
-\nu \frac{\partial^{2} w}{\partial x^{2}}-\nu Q_{1}(t, x) \frac{\partial w}{\partial x}+\frac{\partial w}{\partial t}+\Lambda(t, x) \frac{\partial w}{\partial x} & =L(t, x) w+h(t, x) \\
w(0, x) & =w_{0}(x)
\end{aligned}
$$

then, there exist constants $C=C(T)>1$ and $D$ depending only on $T, T_{0}$, $\|L\|_{L^{\infty}\left(\left[0, T_{0}\right] \times \mathbb{R}, \mathbb{R}^{N}\right)},\left\|\partial_{x} \Lambda\right\|_{L^{\infty}\left(\left[0, T_{0}\right] \times \mathbb{R}, \mathbb{R}^{N}\right)},\left\|Q_{1}\right\|_{L^{\infty}\left(\left[0, T_{0}\right] \times \mathbb{R}_{\mathbb{R}} \mathbb{R}^{N}\right)}$, such that

$\|w\|_{L^{\infty}\left([0, T], L^{2}\left(\mathbb{R}, \mathbb{R}^{N}\right)\right)} \leq C\left(\left\|w_{0}\right\|_{L^{2}\left(\mathbb{R}, \mathbb{R}^{N}\right)}+T\|h\|_{L^{\infty}\left([0, T], L^{2}\left(\mathbb{R}, \mathbb{R}^{N}\right)\right)}\right), \lim _{T \rightarrow 0} C(T)=1$.

$\sqrt{\nu}\left\|\partial_{x} w\right\|_{L^{2}\left([0, T] \times \mathbb{R}, \mathbb{R}^{N}\right)}+\|w\|_{L^{\infty}\left([0, T], L^{2}\left(\mathbb{R}, \mathbb{R}^{N}\right)\right)} \leq D\left(\left\|w_{0}\right\|_{L^{2}\left(\mathbb{R}, \mathbb{R}^{N}\right)}+\|h\|_{L^{2}\left([0, T] \times \mathbb{R}, \mathbb{R}^{N}\right)}\right)$.

We recall this fundamental result.

Theorem 4.2. (Maximum principle) Let $\nu>0, T_{0} \geq T>0, \lambda(t, x) \in$ $C^{1}\left(\left[0, T_{0}\right] \times \mathbb{R}, \mathbb{R}\right), g \in L^{\infty}(] 0, T[\times \mathbb{R}, \mathbb{R}), w_{0} \in L^{\infty}(\mathbb{R}, \mathbb{R})$, and $w(t, x)$ the unique solution in $L^{\infty}\left(\left[0, T_{0}\right] \times \mathbb{R}, \mathbb{R}\right)$ of the following scalar equation with initial data $w_{0}$ :

$$
-\nu \frac{\partial^{2} w}{\partial x^{2}}+\frac{\partial w}{\partial t}+\lambda(t, x) \frac{\partial w}{\partial x}=g(t, x), \quad w(0, x)=w_{0}(x),
$$

then

$$
\|w\|_{L^{\infty}([0, T] \times \mathbb{R}, \mathbb{R})} \leq\left\|w_{0}\right\|_{L^{\infty}(\mathbb{R}, \mathbb{R})}+T\|g\|_{L^{\infty}([0, T] \times \mathbb{R}, \mathbb{R})} .
$$

We are going in next section to use the following interpolation result.

Lemma 4.1. Let $\nu>0, T>0, \lambda(t, x) \in C^{1}([0, T] \times \mathbb{R}, \mathbb{R}), g \in L^{2}([0, T]$ $\times \mathbb{R}, \mathbb{R})$, and $w(t, x)$ the unique solution in $C^{0}\left([0, T], L^{2}\left(\mathbb{R}_{x}, \mathbb{R}\right)\right)$ of the scalar equation, with null initial data:

$$
-\nu \frac{\partial^{2} w}{\partial x^{2}}+\frac{\partial w}{\partial t}+\lambda(t, x) \frac{\partial w}{\partial x}=g(t, x), \quad w(0, x)=0 .
$$

If $\partial_{x} \lambda$ is bounded on $[0, T] \times \mathbb{R}$ then there exists $c_{0}>0$ such that:

$$
\nu^{1 / 4}\|w\|_{L^{\infty}([0, T] \times \mathbb{R}, \mathbb{R})} \leq c_{0}\|g\|_{\left.L^{2}([0, T] \times \mathbb{R}, \mathbb{R})\right)}
$$


Proof. From standard energy estimates (Theorem 4.1), there exists $d_{1}=$ $d_{1}(T)>0$ such that:

$$
\sup _{0 \leq t \leq T}\|w(t, .)\|_{L^{2}\left(\mathbb{R}_{x}\right)} \leq d_{1}\|g\|_{L^{2}([0, T] \times \mathbb{R})} .
$$

We now turn to establish that there exists $d_{2}=d_{2}(T)>0$ such that for each $t \in[0, T]:$

$$
\sqrt{\nu}\left\|\partial_{x} w(t, .)\right\|_{L^{2}\left(\mathbb{R}_{x}\right)} \leq d_{2}\|g\|_{L^{2}([0, T] \times \mathbb{R})} .
$$

Let us begin with smooth $g$ with compact support and conclude by density. Put $z=\partial_{x} w$. Then $z$ satisfies:

$$
-\nu \partial_{x}^{2} z+\partial_{t} z+\lambda \partial_{x} z=-\lambda_{x} z+g_{x}, \quad z(0, x)=0
$$

Following classical computations to get an energy estimate we have:

$$
\begin{aligned}
\int_{\mathbb{R}} \nu\left(\partial_{x} z\right)^{2} d x+\frac{d}{d t} \int_{\mathbb{R}} \frac{z^{2}}{2} d x & =-\int_{\mathbb{R}} \lambda z \partial_{x} z d x-\int_{\mathbb{R}} \lambda_{x} z^{2} d x+\int_{\mathbb{R}} g_{x} z d x \\
& =-\frac{1}{2} \int_{\mathbb{R}} \lambda_{x} z^{2} d x-\int_{\mathbb{R}} g \partial_{x} z d x \\
& \leq \frac{\left\|\lambda_{x}\right\|_{\infty}}{2} \int_{\mathbb{R}} z^{2} d x+\frac{1}{2 \nu} \int_{\mathbb{R}} g^{2} d x+\frac{\nu}{2} \int_{\mathbb{R}}\left(\partial_{x} z\right)^{2} d x \\
\Rightarrow \frac{d}{d t} \int_{\mathbb{R}} z^{2} d x & \leq\left\|\lambda_{x}\right\|_{\infty} \int_{\mathbb{R}} z^{2} d x+\frac{1}{\nu} \int_{\mathbb{R}} g^{2} d x \\
\Rightarrow \int_{\mathbb{R}} z^{2}(t, x) d x & \leq\left\|\lambda_{x}\right\|_{\infty} \int_{0}^{t} \int_{\mathbb{R}} z^{2}(s, x) d s d x+\frac{1}{\nu} \int_{0}^{T} \int_{\mathbb{R}} g^{2} d x,
\end{aligned}
$$

which gives (4.8) by Gronwall Lemma. Then, by the classical interpolation inequality

$$
\|w(t, .)\|_{L^{\infty}\left(\mathbb{R}_{x}, \mathbb{R}\right)}^{2} \leq 2\|w(t, .)\|_{L^{2}\left(\mathbb{R}_{x}, \mathbb{R}\right)}\left\|\partial_{x} w(t, .)\right\|_{L^{2}\left(\mathbb{R}_{x}, \mathbb{R}\right)},
$$

used for each $t$, we conclude the proof.

\section{Proof of the uniform existence}

In this section we prove Proposition 3.1 by a Picard iteration combining energy and $L^{\infty}$ estimates. Fix $T_{0}$ a positive number. Let $M$ be a constant to be chosen later (indeed $M$ will be greater than the profile norm) such that:

$$
\begin{aligned}
& M>M_{0}:=\sup _{0<\varepsilon \leq 1}\left\|v_{0}^{\varepsilon}\right\|_{L^{2} \cap L^{\infty}}, \\
& \text { where }\left\|v_{0}^{\varepsilon}\right\|_{L^{2} \cap L^{\infty}}:=\max \left(\left\|v_{0}^{\varepsilon}\right\|_{L^{2}\left(\mathbb{R}_{x}, \mathbb{R}^{N}\right)},\left\|v_{0}^{\varepsilon}\right\|_{L^{\infty}\left(\mathbb{R}_{x}, \mathbb{R}^{N}\right)}\right) .
\end{aligned}
$$

Let $\mathcal{B}^{\varepsilon}(T)$ be the ball of $C^{0}\left([0, T] ; L^{2}\left(\mathbb{R}, \mathbb{R}^{N}\right)\right) \cap L^{\infty}\left([0, T] \times \mathbb{R}, \mathbb{R}^{N}\right)$ defined by:

$$
\mathcal{B}^{\varepsilon}(T)=\left\{w^{\varepsilon}, w^{\varepsilon}(0, x)=v_{0}^{\varepsilon}(x) \text { and }\left\|w^{\varepsilon}\right\|_{\left.C^{0} L^{2} \cap L^{\infty}\right)} \leq M\right\} .
$$


On $\mathcal{B}^{\varepsilon}(T)$, we define the nonlinear operator $\Pi^{\varepsilon}$ as follows: for any $w^{\varepsilon} \in$ $\mathcal{B}^{\varepsilon}(T), \Pi^{\varepsilon}\left(w^{\varepsilon}\right)$ is the unique solution on $[0, T] \times \mathbb{R}$ of following initial value problem:

$$
\mathbb{L}^{\varepsilon}\left[\Pi^{\varepsilon}\left(w^{\varepsilon}\right)\right]=G^{\varepsilon}\left(t, x, w^{\varepsilon}\right), \quad\left[\Pi^{\varepsilon}\left(w^{\varepsilon}\right)\right](0, x)=v_{0}^{\varepsilon}(x) .
$$

Notice that $v^{\varepsilon}$ is the solution of (4.6), if and only if $\Pi^{\varepsilon}\left(v^{\varepsilon}\right)=v^{\varepsilon}$. We are going to prove that $\Pi^{\varepsilon}$ is a contraction on $\mathcal{B}^{\varepsilon}(T)$ for a positive $T$ with a Lipschitz constant independent of $\varepsilon$.

Lemma 5.1. There exist $T_{1} \in\left(0, T_{0}\right]$ and $\varepsilon_{0} \in(0,1]$ such that for all $\varepsilon \in\left(0, \varepsilon_{0}\right], \Pi^{\varepsilon}\left(\mathcal{B}^{\varepsilon}\left(T_{1}\right)\right) \subset \mathcal{B}^{\varepsilon}\left(T_{1}\right)$.

Proof. We first study $L^{\infty} L^{2}$ stability of $\Pi^{\varepsilon}$.

Since $G^{\varepsilon}(t, x, w)=G(t, x, w)+\varepsilon^{2} Q_{2}(t, x) w$, there exists $c_{1}$ such that for all $w \in \mathcal{B}^{\varepsilon}\left(T_{0}\right)$ :

$$
\left\|G^{\varepsilon}(t, x, w(t, x))\right\|_{L^{\infty} L^{2}} \leq c_{1}\left(1+\|w\|_{L^{\infty} L^{2}}\right) .
$$

By Theorem 4.1 we get:

$$
\begin{aligned}
\left\|\Pi^{\varepsilon}\left(w^{\varepsilon}\right)\right\|_{L^{\infty}\left([0, T] ; L^{2}\right)} & \leq C(T)\left(\left\|w_{0}^{\varepsilon}\right\|_{L^{2}}+T\left\|G^{\varepsilon}\left(t, x, w^{\varepsilon}(t, x)\right)\right\|_{L^{\infty} L^{2}}\right) \\
& \leq C(T)\left(M_{0}+T c_{1}(1+M)\right) .
\end{aligned}
$$

Since $\lim _{T \rightarrow 0} C(T)=1$, there exists $T^{\prime}, 0<T^{\prime} \leq T_{0}$ such that

$$
\left\|\Pi^{\varepsilon}\left(w^{\varepsilon}\right)\right\|_{L^{\infty}\left(\left[0, T^{\prime}\right] ; L^{2}\right)} \leq M .
$$

We now study $L^{\infty}$-stability of $\Pi^{\varepsilon}$.

Let $z^{\varepsilon}=\Pi^{\varepsilon}\left(w^{\varepsilon}\right)$ and $E_{2}:=\sup _{0<\varepsilon \leq 1} \sup _{w^{\varepsilon} \in \mathcal{B}^{\varepsilon}\left(T_{0}\right)}\left\|G^{\varepsilon}\left(t, x, w^{\varepsilon}(t, x)\right)\right\|_{L^{2}\left(\left[0, T_{0}\right] \times \mathbb{R}\right)}<\infty$.

According to Theorem 4.1 we have:

$$
\left\|\varepsilon \partial_{x} z^{\varepsilon}\right\|_{L^{2}\left(\left[0, T_{0}\right] \times \mathbb{R}\right)} \leq D\left(M_{0}+E_{2}\right) .
$$

By linearity, we decompose $z^{\varepsilon}=z_{1}^{\varepsilon}+z_{2}^{\varepsilon}$ in such way that:

$$
\begin{aligned}
& \mathbb{D}^{\varepsilon} z_{1}^{\varepsilon}=G^{\varepsilon}\left(t, x, w^{\varepsilon}\right), \quad z_{1}^{\varepsilon}(0, x)=v_{0}^{\varepsilon}(x), \\
& \mathbb{D}^{\varepsilon} z_{2}^{\varepsilon}=\varepsilon\left(Q_{1}(t, x) \varepsilon \frac{\partial z^{\varepsilon}}{\partial x}\right), \quad z_{2}^{\varepsilon}(0, x)=0 .
\end{aligned}
$$

Since $z_{1}^{\varepsilon}$ system is decoupled, we apply Theorem 4.2 for each component of $z_{1}^{\varepsilon}$ and we have:

$$
\left\|z_{1}^{\varepsilon}\right\|_{L^{\infty}\left(\left[0, T_{0}\right] \times \mathbb{R}\right)} \leq\left\|v_{0}^{\varepsilon}\right\|_{L^{\infty}(\mathbb{R})}+T \sup _{0 \leq t \leq T_{0}, x \in \mathbb{R},|w| \leq M}\left|G^{\varepsilon}(t, x, w)\right| \leq M_{0}+T E_{\infty} .
$$

According to Lemma 4.1 component by component we have:

$$
\left\|z_{2}^{\varepsilon}\right\|_{L^{\infty}([0, T] \times \mathbb{R})} \leq c_{0} \sqrt{\varepsilon}\left(\left\|Q_{1}\right\|_{L^{\infty}\left(\left[0, T_{0}\right] \times \mathbb{R}\right)} D\left(M_{0}+E_{2}\right)\right) .
$$


Then, we can choose $0<T_{1} \leq T^{\prime}$ and $\varepsilon_{0}$ such that for all $\varepsilon \in\left(0, \varepsilon_{0}\right]$ :

$$
\left\|z^{\varepsilon}\right\|_{L^{\infty}\left(\left[0, T_{1}\right] \times \mathbb{R}\right)} \leq\left\|z_{1}^{\varepsilon}\right\|_{L^{\infty}\left(\left[0, T_{1}\right] \times \mathbb{R}\right)}+\left\|z_{2}^{\varepsilon}\right\|_{L^{\infty}\left(\left[0, T_{1}\right] \times \mathbb{R}\right)} \leq M .
$$

Lemma 5.2. There exists $T_{2}$ such that $0<T_{2} \leq T_{1}$ and $\Pi^{\varepsilon}$ is a contraction on $\mathcal{B}^{\varepsilon}\left(T_{2}\right)$ for the $L^{\infty}\left(\left[0, T_{2}\right] ; L^{2}\left(\mathbb{R}, \mathbb{R}^{N}\right)\right)$ norm with a same Lipschitz constant, for all $\varepsilon \in\left(0, \varepsilon_{0}\right]$.

Proof. Let us define $z_{1}^{\varepsilon}=\Pi^{\varepsilon}\left(w_{1}^{\varepsilon}\right), z_{2}^{\varepsilon}=\Pi^{\varepsilon}\left(w_{2}^{\varepsilon}\right), z^{\varepsilon}=z_{1}^{\varepsilon}-z_{2}^{\varepsilon}, w^{\varepsilon}=w_{1}^{\varepsilon}-w_{2}^{\varepsilon}$. $z^{\varepsilon}$ is the solution of the following linear system:

$$
\mathbb{L}^{\varepsilon} z^{\varepsilon}=G^{\varepsilon}\left(t, x, w_{1}^{\varepsilon}\right)-G^{\varepsilon}\left(t, x, w_{2}^{\varepsilon}\right), \quad z^{\varepsilon}(0, x)=0 .
$$

Obviously, there exists a constant $g$ such that

$$
\left\|G^{\varepsilon}\left(t, x, w_{1}^{\varepsilon}\right)-G^{\varepsilon}\left(t, x, w_{2}^{\varepsilon}\right)\right\|_{L^{\infty}\left(\left[0, T_{0}\right] ; L^{2}\right)} \leq g\left\|\left|w^{\varepsilon}\right|\right\|_{L^{\infty}\left(\left[0, T_{0}\right] ; L^{2}\right)},
$$

then $\left\|z^{\varepsilon}\right\|_{L^{\infty}\left(\left[0, T_{0}\right] ; L^{2}\right)} \leq C T g\left\|w^{\varepsilon}\right\|_{L^{\infty}\left(\left[0, T_{0}\right] ; L^{2}\right)}$ so we can choose $0<T_{2} \leq T_{1}$ such that $\Pi^{\varepsilon}$ is a contraction for the $L^{\infty} L^{2}$ norm on $\mathcal{B}^{\varepsilon}\left(T_{2}\right)$ with a Lipschitz constant independent of $\varepsilon$.

Proof of Proposition 3.1. Let $v_{n+1}^{\varepsilon}=\Pi^{\varepsilon}\left(v_{n}^{\varepsilon}\right), n \in \mathbb{N}$ and $v_{n=0}^{\varepsilon}(t, x)=$ $v_{0}^{\varepsilon}(x)$. Lemmas 5.1 and 5.2 imply the uniform existence of $\left(v^{\varepsilon}\right)_{0<\varepsilon \leq \varepsilon_{0}}$, on $\left[0, T_{2}\right] \times \mathbb{R}$ and $\lim _{n \rightarrow+\infty} v_{n}^{\varepsilon}=v^{\varepsilon}$ in $C^{0} L^{2}$. Then $v^{\varepsilon}$ also belongs to $\mathcal{B}^{\varepsilon}\left(T_{2}\right)$.

\section{On profile equations}

Lemma 6.1. ( $L^{\infty}$ and $L^{2}$ estimates for linear scalar profile equation) Let $j$ be fixed, $V_{0 j} \in L^{2}\left(\mathbb{R} ; C_{a p}^{0}\left(\Theta^{0}, \mathbb{R}\right)\right) \cap L^{\infty}\left(\mathbb{R} \times \Theta^{0}, \mathbb{R}\right), f_{j} \in C^{0}([0, T]$; $\left.L^{2}\left(\mathbb{R} ; C_{a p}^{0}\left(\Theta^{j}, \mathbb{R}\right)\right)\right) \cap L^{\infty}\left([0, T] \times \mathbb{R} \times \Theta_{j}, \mathbb{R}\right)$, and $V_{j}\left(t, x, \theta_{j}\right)$ be the unique solution of the scalar equation, with initial data:

$$
-\mathcal{D}_{j}^{2} V_{j}+X_{j} V_{j}=f_{j}, \quad V_{j}\left(0, x, \theta_{j}\right)=V_{0 j}\left(0, x, \theta_{j}\right) .
$$

Then, there exists $C$ such that:

$$
\begin{aligned}
\left\|V_{j}\right\|_{L^{\infty}\left([0, T] \times \mathbb{R} \times \Theta_{j}, \mathbb{R}\right)} & \leq\left\|V_{0 j}\right\|_{L^{\infty}\left(\mathbb{R} \times \Theta^{0}, \mathbb{R}\right)}+T\left\|f_{j}\right\|_{L^{\infty}\left([0, T] \times \mathbb{R} \times \Theta_{j}, \mathbb{R}\right)}, \\
\left\|V_{j}\right\|_{C^{0}\left([0, T], L^{2}\left(\mathbb{R} ; C_{a p}^{0}\left(\Theta_{j}\right)\right)\right)} & \leq C\left(\left\|V_{0 j}\right\|_{L^{2}\left(\mathbb{R} ; C_{a p}^{0}\left(\Theta^{0}\right)\right)}+T\left\|f_{j}\right\|_{C^{0}\left([0, T], L^{2}\left(\mathbb{R} ; C_{a p}^{0}(\Theta)\right)\right)}\right) .
\end{aligned}
$$

Proof. The first inequality is the classical maximum principle. Therefore, we have only to prove the second one. Let $w_{j}$ be the solution of:

$$
\begin{array}{r}
-\mathcal{D}_{j}^{2} w_{j}+X_{j} w_{j}=\bar{f}_{j}=\left\|f_{j}(t, x, .)\right\|_{L^{\infty}(\Theta)}, \\
w_{j}\left(0, x, \theta_{j}\right)=\bar{V}_{0 j}=\left\|V_{0 j}(0, x, .)\right\|_{L^{\infty}\left(\Theta^{j}\right)} .
\end{array}
$$


By maximum principle $\left|V_{j}\left(t, x, \theta_{j}\right)\right| \leq\left|w_{j}\left(t, x, \theta_{j}\right)\right|$ and, by uniqueness, $w_{j}$ does not depend of $\theta$. In fact, $w_{j}$ is solution of: $X_{j} w_{j}=\bar{f}_{j}, w_{j}\left(0, x, \theta_{j}\right)=\bar{V}_{0 j}$. It is a linear hyperbolic equation, so the classical energy inequality for $w_{j}$ gives us the result.

Now we are able to prove Proposition 3.2: By a standard Picard iteration, $V^{0}(t, x, \theta)=V_{0}(x, \theta)$, and, for $n \in \mathbb{N}, j=1, \ldots, N, V_{j}^{n+1}$ is given by:

$$
-\mathcal{D}_{j}^{2} V_{j}^{n+1}+X_{j} V_{j}^{n+1}=\mathbb{E}_{j} G_{j}\left(t, x, V^{n}\right), \quad V_{j}^{n+1}\left(0, x, \theta_{j}\right)=V_{0, j}\left(x, \theta_{j}\right) .
$$

Thanks to Lemma 6.1 , and since $\left\|\mathbb{E}_{j}\right\|=1$ we get classically a contraction for small positive $T$.

A straightforward computation gives the following useful Lemma:

Lemma 6.2. (Periodic exponential solution) Let $j$ be fixed, $T$ positive, $\alpha \in \Theta^{*}, b_{\alpha}(t, x) \in C^{0}\left([0, T] ; L^{2}(\mathbb{R}, \mathbb{R})\right), a_{\alpha}^{0}(t, x) \in L^{2}(\mathbb{R}, \mathbb{R})$. Let $V$ be the unique solution of:

$$
-\mathcal{D}_{j}^{2} V+X_{j} V=b_{\alpha}(t, x) \exp (i \alpha \cdot \theta), \quad V(0, x, \theta)=a_{\alpha}^{0}(x) \exp (i \alpha \cdot \theta) .
$$

Then $V(t, x, \theta)=a_{\alpha}(t, x) \exp (i \alpha \cdot \theta)$, where $a_{\alpha}$ is the solution of:

$$
X_{j} a_{\alpha}+\left|\alpha . \partial_{x} \phi_{j}\right|^{2} a_{\alpha}=b_{\alpha}(t, x), \quad a_{\alpha}(0, x)=a_{\alpha}^{0}(x) .
$$

We then can compute Fourier expansion of $V$. Indeed $a_{\alpha}$ has the same smoothness as $a_{\alpha}^{0}$ and $b_{\alpha}$. Furthermore, if data are compactly supported w.r.t. $x$, so are $a_{\alpha}$ and $V$.

\section{The linear scalar case}

In this section, we validate the geometric optics expansion for the linear scalar case. The last result comes in useful to prove the main result of this paper.

Lemma 7.1. Let $0<\varepsilon \leq 1, h \in L^{2}\left(\mathbb{R} ; C_{a p}^{0}\left(\Theta^{0}, \mathbb{R}\right)\right)$, and $j \in\{1, \cdots, N\}$. Let $u^{\varepsilon}$ and $U$ be the unique solutions of equations $(7.1),(7.2)$ on $[0, T] \times \mathbb{R}$,

$$
\begin{aligned}
-\varepsilon^{2} \partial_{x}^{2} u^{\varepsilon}+X_{j} u^{\varepsilon} & =0, & u^{\varepsilon}(0, x)=h\left(x, \frac{\varphi^{0}}{\varepsilon}\right), \\
-\mathcal{D}_{j}^{2} U+X_{j} U & =0, & U\left(0, x, \theta_{j}\right)=h\left(x, \theta_{j}\right),
\end{aligned}
$$

then $u^{\varepsilon}(t, x)=U\left(t, x, \frac{\varphi_{j}}{\varepsilon}\right)+O(\varepsilon)$ in $L^{\infty}\left([0, T] ; L^{2}(\mathbb{R}, \mathbb{R})\right)$ 
Proof. First, we assume that $h \in C_{c}^{\infty}\left(\mathbb{R}_{x} ; C_{a p}^{\infty}\left(\Theta^{0}, \mathbb{R}\right)\right)$, where the subscript $c$ means that $h$ has compact support with respect to $x$. Let

$$
v^{\varepsilon}(t, x)=U\left(t, x, \frac{\varphi_{j}(t, x)}{\varepsilon}\right) .
$$

Then, a direct computation give us with $S^{\varepsilon}(t, x):=-\varepsilon^{2} \partial_{x}^{2} U-\varepsilon\left(\partial_{x} \mathcal{D}_{j}\right) U=$ $\mathrm{O}(\varepsilon) \in L^{\infty}\left([0, T] ; L^{2}(\mathbb{R}, \mathbb{R})\right)$ :

$$
-\varepsilon^{2} \partial_{x}^{2} v^{\varepsilon}+X_{j} v^{\varepsilon}=S^{\varepsilon}-\mathcal{D}_{j}^{2} U+X_{j} U=S^{\varepsilon}, \quad v^{\varepsilon}(0, x)=u^{\varepsilon}(0, x) .
$$

Since $-\varepsilon^{2} \partial_{x}^{2} u^{\varepsilon}+X_{j} u^{\varepsilon}=0$, we can apply energy estimate to $u^{\varepsilon}-v^{\varepsilon}$ to conclude this smooth case. We finish the proof using density of $C_{c}^{\infty}\left(\mathbb{R}_{x} ; C_{a p}^{\infty}\left(\Theta^{0}, \mathbb{R}\right)\right)$ in $L^{2}\left(\mathbb{R} ; C_{a p}^{0}\left(\Theta^{0}, \mathbb{R}\right)\right)$.

We recall and slightly extend to the case of an unbounded domain with a linear term, the statement of non stationary phases Lemma from [13]:

Lemma 7.2. Let $0<\varepsilon \leq 1, a \in C^{0}\left([0, T] ; L^{2}(\mathbb{R}, \mathbb{R})\right), b \in L^{\infty}([0, T] \times \mathbb{R}, \mathbb{R})$, $\psi \in C^{\infty}([0, T] \times \mathbb{R}, \mathbb{R})$ and $j \in\{1, \cdots, N\}$. Assume that $\psi$ is transverse to $X_{j}$ i.e. $X_{j} \psi \neq 0$ a.e., and $u^{\varepsilon}$ is the unique solution of $(7.3)$ on $[0, T] \times \mathbb{R}$,

$$
X_{j} u^{\varepsilon}+b(t, x) u^{\varepsilon}=a(t, x) \exp \left(i \frac{\psi(t, x)}{\varepsilon}\right), \quad u^{\varepsilon}(0, x)=0,
$$

then $\lim _{\varepsilon \rightarrow 0} u^{\varepsilon}(t, x)=0$ in $L^{\infty}\left([0, T] ; L^{2}\left(\mathbb{R}, \mathbb{R}^{N}\right)\right)$.

We now apply the previous Lemma to a weakly parabolic equation.

Lemma 7.3. Let $0<\varepsilon \leq 1, a \in C^{0}\left([0, T] ; L^{2}(\mathbb{R}, \mathbb{R})\right), j \in\{1, \cdots, N\}$. Let $u^{\varepsilon}, U$ the unique solution of the following equations on $[0, T] \times \mathbb{R}$

$$
\begin{aligned}
-\varepsilon^{2} \partial_{x}^{2} u^{\varepsilon}+X_{j} u^{\varepsilon} & =a(t, x) \exp \left(i \alpha \cdot \frac{\varphi(t, x)}{\varepsilon}\right), & u^{\varepsilon}(0, x) & =0, \\
-\mathcal{D}_{j}^{2} U+X_{j} U & =a(t, x) \mathbb{E}_{j}(\exp (i \alpha \cdot \theta)), & & U\left(0, x, \theta_{j}\right)=0,
\end{aligned}
$$

then $\lim _{\varepsilon \rightarrow 0}\left[u^{\varepsilon}(t, x)-U\left(t, x, \frac{\varphi_{j}(t, x)}{\varepsilon}\right)\right]=0$ in $L^{\infty}\left([0, T] ; L^{2}\left(\mathbb{R}, \mathbb{R}^{N}\right)\right)$.

Proof. First, we assume that $\left.a \in C_{c}^{2}([0, T] \times \mathbb{R}, \mathbb{R})\right)$. We then extend the result to more general data by density.

Let $\psi: \equiv \alpha . \varphi$. By transversality assumptions on phase $\varphi$, we have only two cases: 
1. $\boldsymbol{X}_{\boldsymbol{j}} \boldsymbol{\psi} \equiv \mathbf{0}$ : then we have $\mathbb{E}_{j}(\exp (i \alpha \cdot \theta))=\exp (i \alpha . \theta)$. As in Lemma 7.1 proof, let us consider

$$
v^{\varepsilon}(t, x):=U\left(t, x, \frac{\varphi_{j}(t, x)}{\varepsilon}\right)
$$

then we have:

$$
-\varepsilon^{2} \partial_{x}^{2} v^{\varepsilon}+X_{j} v^{\varepsilon}=a(t, x) \exp \left(i \alpha \cdot \frac{\varphi(t, x)}{\varepsilon}\right)+O(\varepsilon), \quad v^{\varepsilon}(0, x)=0,
$$

and we conclude this case by applying an energy estimate to $u^{\varepsilon}-v^{\varepsilon}$.

2. $\boldsymbol{\psi}$ is transverse to $\boldsymbol{X}_{\boldsymbol{j}}$ : then we have $\mathbb{E}_{j} \exp (i \alpha . \theta) \equiv 0$ and $U \equiv 0$. Let $w^{\varepsilon}$ be the solution of

$$
X_{j} w^{\varepsilon}=a(t, x) \exp \left(i \alpha \cdot \frac{\varphi(t, x)}{\varepsilon}\right), \quad w^{\varepsilon}(0, x)=0 .
$$

From Lemma 7.2 we get $w^{\varepsilon} \rightarrow 0$ in $L_{t}^{\infty} L_{x}^{2}$. In the same way, we are going to see that $\varepsilon \partial_{x} w^{\varepsilon}$ and $\varepsilon^{2} \partial_{x}^{2} w^{\varepsilon}$ also converge towards 0 in $L^{2}$. First, we take the derivative of (7.4) with respect to $\varepsilon \partial_{x}$ and then with respect to $\varepsilon^{2} \partial_{x}^{2}$ to get:

$$
X_{j} z^{\varepsilon}+b z^{\varepsilon}=\beta \exp \left(i \frac{\psi}{\varepsilon}\right)+\mathrm{O}(\varepsilon), \quad z^{\varepsilon}(0, x)=0,
$$

first with $z^{\varepsilon}:=\varepsilon \partial_{x} w^{\varepsilon}$, and after with $z^{\varepsilon}:=\varepsilon^{2} \partial_{x}^{2} w^{\varepsilon}$. Then, we conclude with Lemma 7.2. More precisely, we obtain the following equations:

$$
\begin{aligned}
& X_{j}\left(\varepsilon \partial_{x} w^{\varepsilon}\right)+\left(\partial_{x} \lambda_{j}\right)\left(\varepsilon \partial_{x} w^{\varepsilon}\right)=i a \partial_{x} \psi \exp \left(i \frac{\psi}{\varepsilon}\right)+\varepsilon \times\left(\partial_{x} a \exp \left(i \frac{\psi}{\varepsilon}\right)\right) \\
& X_{j}\left(\varepsilon^{2} \partial_{x}^{2} v^{\varepsilon}\right)+\left(\partial_{x} \lambda_{j}\right)\left(\varepsilon^{2} \partial_{x}^{2} w^{\varepsilon}\right)=i a\left(\partial_{x} \psi\right)^{2} \exp \left(i \frac{\psi}{\varepsilon}\right)+\mathrm{O}(\varepsilon), \text { where } \\
& \left.\mathrm{O}(\varepsilon)=\varepsilon\left[\left(i a \partial_{x}^{2} \psi\right)+2\left(\partial_{x} a\right)\left(\partial_{x} \psi\right)+\varepsilon \partial_{x}^{2} a\right) \exp (i \psi / \varepsilon)-\left(\partial_{x}^{2} \lambda_{j}\right)\left(\varepsilon \partial_{x} w^{\varepsilon}\right)\right] .
\end{aligned}
$$

Now, let $R^{\varepsilon}:=u^{\varepsilon}-w^{\varepsilon}$. We can conclude by using energy estimate since $R^{\varepsilon}$ is the solution of:

$$
-\varepsilon^{2} \partial_{x}^{2} R^{\varepsilon}+X_{j} R^{\varepsilon}=\varepsilon^{2} \partial_{x}^{2} w^{\varepsilon}, \quad w^{\varepsilon}(0, x)=0 .
$$

Proposition 7.1. Let $0<\varepsilon \leq 1$,

$$
h \in L^{2}\left(\mathbb{R} ; C_{a p}^{0}\left(\Theta^{0}, \mathbb{R}\right)\right), \quad f \in C^{0}\left([O, T] ; L^{2}\left(\mathbb{R} ; C_{a p}^{0}(\Theta, \mathbb{R})\right)\right),
$$

and let $u^{\varepsilon}, U$ be the unique solutions of the following equations on $[0, T]$

$$
\begin{aligned}
-\varepsilon^{2} \partial_{x}^{2} u^{\varepsilon}+X_{j} u^{\varepsilon} & =f\left(t, x, \frac{\varphi}{\varepsilon}\right), & & u^{\varepsilon}(0, x)=h\left(x, \frac{\varphi^{0}}{\varepsilon}\right) \\
-\mathcal{D}_{j}^{2} U+X_{j} U & =\mathbb{E}_{j} f(t, x, \theta), & & U\left(0, x, \theta_{j}\right)=h\left(x, \theta_{j}\right)
\end{aligned}
$$


Then

$$
\lim _{\varepsilon \rightarrow 0}\left[u^{\varepsilon}(t, x)-U\left(t, x, \frac{\varphi_{j}(t, x)}{\varepsilon}\right)\right]=0 \text { in } L^{\infty}\left([0, T] ; L^{2}\left(\mathbb{R}, \mathbb{R}^{N}\right)\right) .
$$

Proof. By linearity of the previous P.D.E. we can decompose $u^{\varepsilon}=u_{1}^{\varepsilon}+u_{2}^{\varepsilon}$. We make an analogous decomposition for $U . u_{1}^{\varepsilon}, u_{2}^{\varepsilon}, U_{1}, U_{2}$ are solutions of following equations

$$
\begin{aligned}
-\varepsilon^{2} \partial_{x}^{2} u_{1}^{\varepsilon}+X_{j} u_{1}^{\varepsilon} & =0, & u_{1}^{\varepsilon}(0, x)=h\left(x, \frac{\varphi^{0}}{\varepsilon}\right), \\
-\varepsilon^{2} \partial_{x}^{2} u_{2}^{\varepsilon}+X_{j} u_{2}^{\varepsilon} & =f\left(t, x, \frac{\varphi}{\varepsilon}\right), & u_{2}^{\varepsilon}(0, x)=0, \\
-\mathcal{D}_{j}^{2} U_{1}+X_{j} U & =0, & U_{1}(0, x, \theta)=h(x, \theta), \\
-\mathcal{D}_{j}^{2} U_{2}+X_{j} U & =\mathbb{E}_{j} f(t, x, \theta), & U_{2}(0, x, \theta)=0 .
\end{aligned}
$$

By Lemma 7.1, we obtain $u_{1}^{\varepsilon}=U_{1}(t, x, \varphi / \varepsilon)+\mathrm{O}(\varepsilon)$. For $u_{2}^{\varepsilon}$ we use the density of trigonometric polynomials, as in [13]. We then replace $f$ by a trigonometric polynomial, and, by linearity, we can use Lemma 7.3. The proof is thus complete.

\section{Proof of geometric optics expansion}

The proof is based on the following observation as in [14]. For a contraction mapping $\Pi$ with a Lipschitz constant $\rho$ and the fixed point $u$, we recall a classical a posteriori estimate:

$$
\|u-v\| \leq \frac{1}{1-\rho}\|\Pi(v)-v\|
$$

Let $w^{\varepsilon}(t, x)=V\left(t, x, \frac{\varphi(t, x)}{\varepsilon}\right)$. With stationary and non stationary phase Lemmas we are going to prove that $\Pi^{\varepsilon}\left(w^{\varepsilon}\right)=w^{\varepsilon}+o(1)$. Since $\left(\Pi^{\varepsilon}\right)_{0<\varepsilon \leq \varepsilon_{0}}$ is a family of contraction with same Lipschitz constant we have: $v^{\varepsilon}=w^{\varepsilon}+o(1)$.

Precisely, we use notations from Section 5. Let $T_{3}$ satisfying Proposition 3.2: a time existence of the profile.

Fix $M>\|V\|_{L^{\infty}\left(\left[0, T_{3}\right] ; L^{2}\left(\mathbb{R}: C_{a p}^{0}\left(\Theta, \mathbb{R}^{N}\right)\right)\right)}+\|V\|_{L^{\infty}\left(\left[0, T_{3}\right] \times \mathbb{R} \times \Theta, \mathbb{R}^{N}\right)}$. We now choose $T_{4}$ such that $v^{\varepsilon}, V$ live on $\left[0, T_{4}\right], \Pi^{\varepsilon}\left(\mathcal{B}^{\varepsilon}\left(T_{4}\right)\right) \subset \mathcal{B}^{\varepsilon}\left(T_{4}\right)$, and $\Pi^{\varepsilon}$ are a uniform $L^{\infty} L^{2}$ contraction on $\mathcal{B}^{\varepsilon}\left(T_{4}\right)$.

First $w^{\varepsilon}$ belongs to $\mathcal{B}^{\varepsilon}\left(T_{4}\right)$. Let $z^{\varepsilon}=\Pi^{\varepsilon}\left(w^{\varepsilon}\right)$ and $j$ fixed. Notice that $z^{\varepsilon}$ belongs to $\mathcal{B}^{\varepsilon}\left(T_{4}\right)$. $z_{j}^{\varepsilon}$ satisfies the following equation:

$$
-\varepsilon^{2} \partial_{x}^{2} z_{j}^{\varepsilon}+X_{j} z_{j}^{\varepsilon}=G_{j}\left(t, x, V\left(t, x, \frac{\varphi(t, x)}{\varepsilon}\right)\right)+r_{j}^{\varepsilon}
$$


where $r^{\varepsilon}=\varepsilon^{2} Q_{2} w^{\varepsilon}+\varepsilon^{2} Q_{1} \partial_{x} z^{\varepsilon}=O(\varepsilon)$ in $L^{\infty} L^{2}$. Neglecting $r^{\varepsilon}$, and according to Proposition 7.1, we then have $z_{j}^{\varepsilon}(t, x)=W_{j}\left(t, x, \frac{\varphi_{j}}{\varepsilon}\right)+o(1)$, where $W_{j}$ is the solution of

$$
\left(-\mathcal{D}_{j}^{2}+X_{j}\right) U=\mathbb{E}_{j} G_{j}(t, x, V(t, x, \theta)) \text { and } W_{j}(0, ., .)=V_{j}(0, ., .) .
$$

Therefore $W_{j}$ is exactly $V_{j}, z_{j}^{\varepsilon}=w_{j}^{\varepsilon}+o(1)$ and:

$$
\Pi^{\varepsilon}\left(w^{\varepsilon}\right)=w^{\varepsilon}+o(1) \quad \text { in } \quad L^{\infty}\left(\left[0, T_{4}\right] ; L^{2}\left(\mathbb{R}, \mathbb{R}^{N}\right)\right)
$$

Since $\Pi^{\varepsilon}$ is a $L^{\infty}\left(\left[0, T_{4}\right] ; L^{2}\left(\mathbb{R}, \mathbb{R}^{N}\right)\right)$ contraction on $\mathcal{B}^{\varepsilon}\left(T_{4}\right)$ with a Lipschitz constant independent of $\varepsilon \in\left(0, \varepsilon_{0}\right]$, we have

$$
v^{\varepsilon}=w^{\varepsilon}+o(1) \quad \text { in } \quad L^{\infty}\left(\left[0, T_{4}\right] ; L^{2}\left(\mathbb{R}, \mathbb{R}^{N}\right)\right),
$$

which completes the proof of Theorem 3.1.

\section{Comparisons with the inviscid case}

Let us first investigate the standard non stationary phase Lemma. Could this Lemma be improved by the presence of small viscosity? Unfortunately the answer is no as can be seen from the following simple example.

$$
\begin{array}{rlrl}
-\varepsilon^{2} \partial_{x}^{2} u^{\varepsilon}+\partial_{t} u^{\varepsilon} & =\exp (i \phi(t, x) / \varepsilon), & & u^{\varepsilon}(0, x)=0, \\
\partial_{t} v^{\varepsilon}=\exp (i \phi(t, x) / \varepsilon), & & v^{\varepsilon}(0, x)=0,
\end{array}
$$

with $X=\partial_{t}+0 \times \partial_{x}=\partial_{t}$.

Assume $X \phi \neq 0$ a.e., for instance with $\phi(t, x)=t$, viscosity plays no role since $u^{\varepsilon}(t, x)=v^{\varepsilon}(t, x)=i \varepsilon(1-\exp (i t / \varepsilon))=\mathrm{O}(\varepsilon)$.

Furthermore, if $\phi(t, x)=\psi(t)$, we always have $u^{\varepsilon}(t, x)=v^{\varepsilon}(t, x)$. If $\psi^{\prime}$ vanishes on a discrete set, we have no rate of convergence. For instance if $\psi(t) \sim t^{\alpha}$ when $t$ goes to 0 and $\alpha>1$, we have $u^{\varepsilon}(t, x) \sim C \varepsilon^{1 / \alpha}$ with $C \neq 0$. We cannot expect any correctors for multiphase expansions with such general transversality conditions on phases. This construction follows [13, Remark 1.2, p. 114-115].

The new feature in our paper is to get and validate parabolic equations for profiles. These equations are only related to phases transported by the hyperbolic operator $\partial_{t}+A(t, x) \partial_{x}$. For instance, solutions of (9.1),(9.2) with $\phi(t, x)=x$, are $u^{\varepsilon}(t, x)=\left(1-e^{-t}\right) \exp (i x / \varepsilon), v^{\varepsilon}(t, x)=t \exp (i x / \varepsilon)$.

Profiles satisfy the following equations:

$$
\begin{aligned}
& -\partial_{\theta}^{2} U+\partial_{t} U=\exp (i \theta), \quad U(0, x, \theta)=0, \\
& \partial_{t} V=\exp (i \theta), \quad V(0, x, \theta)=0 .
\end{aligned}
$$


The viscous profile is then given by $U(t, x, \theta)=\left(1-e^{-t}\right) e^{i \theta}$, and the hyperbolic profile is given by $V(t, x, \theta)=t e^{i \theta}$. Except for small time, $u^{\varepsilon}$ and $v^{\varepsilon}$ have different behaviors.

We conclude this section with a few remarks on large time behavior. If $\left(u^{\varepsilon}\right)_{0<\varepsilon \leq 1}$ and $U$ are bounded in $L^{\infty}\left([0, T], L^{2}\left(\mathbb{R}_{x}\right)\right) \cap L^{\infty}\left([0, T] \times \mathbb{R}_{x}\right)$ then geometric expansion stated in theorem 3.1 is still valid on $[0, T]$. In general, however the maximal existence time of the family of exact solutions is not bounded from below by the existence time for the profile. Indeed, to have such result we have to validate the WKB expansion in $L^{\infty}\left([0, T] \times \mathbb{R}_{x}\right)$. For this purpose we have to strengthen transversality assumptions on phases and smoothness of data and solutions. For a fuller treatment of existence times in the inviscid case we refer the reader to [13].

\section{References}

[1] Cheverry, C.: Propagation of oscillations in real vanishing viscosity limit. Comm. Math. Phys. 247 (2004), no. 3, 655-695.

[2] Choquet-Bruhat, Y.: Ondes asymptotiques et approchées pour des systèmes d'équations aux dérivées partielles non linéaires. J. Math. Pures Appl. (9) 48 (1969), 117-158.

[3] Corduneanu, C.: Almost periodic functions. Interscience Tracts in Pure and Applied Mathematics 22. Interscience Publishers, John Wiley, New York-London-Sydney, 1968.

[4] Chen, G.-Q., Junca, S. and Rascle, M.: Validity of Nonlinear Geometric Optics for Entropy Solutions of Multidimensional Scalar Conservation Laws. J. Differential Equations 222 (2006), no. 2, 439-475.

[5] DiPerna, R. J. And Majda, A.: The validity of nonlinear geometric optics for weak solutions of conservation laws. Comm. Math. Phys. 98 (1985), no. 3, 313-347.

[6] Dumas, E.: About nonlinear geometric optics. Bol. Soc. Esp. Mat. Apl. 35 (2006), 7-41.

[7] Gallaire, F., Gérard-Varet, D. and Rousset, F.: Three-dimensional instability of planar flows. Arch. Ration. Mech. Anal. 186 (2007), no. $3,423-475$.

[8] Gallagher, I.: Applications of Schochet's methods to parabolic equations. J. Math. Pures Appl. (9) 77 (1998), no. 10, 989-1054.

[9] Gues, O.: Ondes solitaires engendrées par interaction d'ondes oscillantes non linéaires. J. Math. Pures Appl. (9) 74 (1995), no. 3, 199-252.

[10] GuEs, O.: Vanishing viscosity boundary layers for nonlinear hyperbolic systems. In Nonlinear theory of generalized functions (Vienna, 1997), 75-84. Chapman \& Hall/CRC Res. Notes Math. 401. Chapman \& Hall/CRC, Boca Raton, FL, 1999. 
[11] Hunter, J. K. And Keller, J. B.: Weakly nonlinear high frequency waves. Comm. Pure Appl. Math. 36 (1983), no. 5, 547-569.

[12] Hunter, J.K., Majda, A. And Rosales, R.: Resonantly interacting, weakly nonlinear hyperbolic waves. II. Several space variables. Stud. Appl. Math. 75 (1986), no. 3, 187-226.

[13] Joly, J.-L., Métivier, G. And Rauch, J.: Resonant one-dimensional nonlinear geometric optics. J. Funct. Anal. 114 (1993), no. 1, 106-231.

[14] JuncA, S.: Réflexions d'oscillations monodimensionnelles. Comm. Partial Differential Equations 23 (1998), no. 3-4, 727-759.

[15] JuncA, S.: A two-scale convergence result for a nonlinear scalar conservation law in one space variable. Asymptot. Anal. 17 (1998), no. 3, 221-238.

[16] Kalyakin, L. A.: Long-wave asymptotics. Integrable equations as the asymptotic limit of nonlinear systems. Uspekhi Mat. Nauk 44 (1989), no. 1(265), 5-34, 247; translation in Russian Math. Surveys 44 (1989), no. $1,3-42$.

[17] Lax, P. D.: Asymptotic solutions of oscillatory initial value problems. Duke Math. J. 24 (1957), 627-646.

[18] Majda, A. And Rosales, R.: Resonantly interacting weakly nonlinear hyperbolic waves. I. A single space variable. Stud. Appl. Math. 71 (1984), no. 2, 149-179.

[19] Protter, M.H. And Weinberger, H. F.: Maximum principles in differential equations. Springer-Verlag, New York, 1984.

[20] Williams, M.: Resonant reflection of multidimensional semilinear oscillations. Comm. Partial Differential Equations 18 (1993), no. 11, 1901-1959.

[21] Williams, M.: Nonlinear geometric optics for reflecting and glancing oscillations. In Singularities and oscillations (Minneapolis, MN, 1994/1995), 137-151. IMA Vol. Math. Appl. 91. Springer, New York, 1997.

[22] Zeidler, E.: Nonlinear Functional Analysis and its Applications. SpringerVerlag, New York, 1993.

Recibido: 26 de octubre de 2006

Stéphane Junca

IUFM \& Université de Nice Labo. J.A.D., UMR CNRS 6621

Parc Valrose

F-06108, Nice, France junca@math.unice.fr

The author wishes to express his thanks to Olivier Gues for suggesting the problem and for many stimulating conversations, and to Jean-François Collet for some useful remarks. 